\title{
Discontinuous Galerkin Time-Domain Analysis of Power/Ground Plate Pairs with Wave Port Excitation
}

\author{
Ping Li and Li Jun Jiang \\ Department of Electrical and \\ Electronic Engineering \\ The University of Hong Kong \\ Hong Kong \\ Email: liping@eee.hku.hk, jianglj@hku.hk
}

\author{
Hakan Bağc1 \\ Division of Computer, Electrical, \\ and Mathematical Sciences and Engineering \\ King Abdullah University of Science and Technology, \\ Thuwal, 23955-6900, Saudi Arabia \\ Email: hakan.bagci@kaust.edu.sa
}

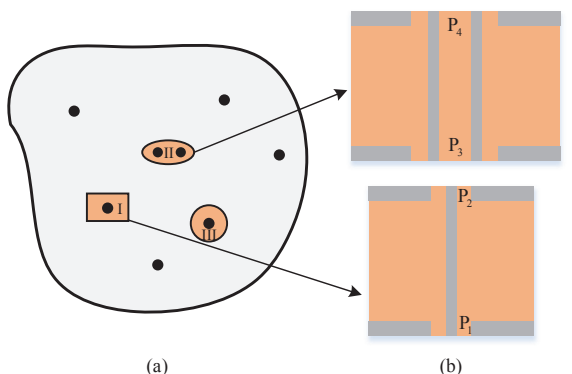

Fig. 1. Geometrical illustration of a power-ground plate-pair with various types of vias in different anti-pads. (a) The Top view of the plate-pair. (b) The side view of via I and II.

can be utilized to approximate the solution in a local manner. Consequently, DGTD integrates the advantages of finite volume method (FVM) and FEM.

In order to enforce proper wave port excitation over the anti-pad, the magnetic current source used in this work is expanded by the eigen-modes supported by the anti-pad. In this way, only the modes of interests can be used for excitation, which makes the proposed algorithm more flexible in studying the coupling and conversion between different modes, etc. Usually, the anti-pads are in an irregular shape or have many via barrels. In this situation, the eigen-modes cannot be derived analytically but must be solved through numerical techniques. By leveraging the orthogonal property of eigen-modes, the incident, transmitted and reflected mode expansion coefficients in the time-domain can be accurately extracted. Then, the Sparameters are able to be obtained based on the pre-calculated mode coefficients.

\section{Magnetic Current Based Wave Port Excitation AND S-PARAMETER EXTRACTION}

In order to implement proper excitation over the anti-pad region, in this work, the magnetic surface current approximated by the superposition of different electric eigen-modes of the anti-pad is applied as the source. For regular concentric vias, the analytical expressions of the eigen-modes supported by the anti-pad are available, thus it is ready and convenient to apply the excitation. However, for arbitrarily shaped antipads 
such as rectangular or elliptic pads, the corresponding eigenmodes are not analytically available, thus they have to be numerically calculated. As shown in Fig. 1, we have vias with arbitrarily shaped anti-pads in an irregular plate-pair. It can be noted that there are three typical vias: i) traditional via with a circular anti-pad (via III); ii) normal via located in the center of a rectangular anti-pad (via I); (iii) differential viapairs placed in an elliptic anti-pad (via II). For via III, the analytical solutions of the eigen-modes are well-known. On the other side, numerical eigen-modes are required for via I and II.

At the wave port $\mathrm{P}_{i}(i=1, \cdots, 4)$, the tangential components of the electric and magnetic fields can be approximated by the summation of the eigen-modes. Namely,

$$
\begin{aligned}
& \mathbf{E}_{t}(\mathbf{r}, \omega)=a_{0}^{\mathrm{TEM}} \mathbf{e}_{0}^{\mathrm{TEM}}+\sum_{n=1}^{\infty} a_{n}^{\mathrm{TE}} \mathbf{e}_{n}^{\mathrm{TE}}+\sum_{n=1}^{\infty} a_{n}^{\mathrm{TM}} \mathbf{e}_{n}^{\mathrm{TM}} \\
& \mathbf{H}_{t}(\mathbf{r}, \omega)=b_{0}^{\mathrm{TEM}} \mathbf{h}_{0}^{\mathrm{TEM}}+\sum_{n=1}^{\infty} b_{n}^{\mathrm{TE}} \mathbf{h}_{n}^{\mathrm{TE}}+\sum_{n=1}^{\infty} b_{n}^{\mathrm{TM}} \mathbf{h}_{n}^{\mathrm{TM}},
\end{aligned}
$$

where $\mathbf{e}_{n}$ and $\mathbf{h}_{n}$ denote the electric and magnetic eigenmodes, respectively; $a_{n}$ and $b_{n}$ are the corresponding mode expansion coefficients.

To apply the magnetic current excitation over the wave port (or the anti-pad), the electric eigen-modes should be calculated, then the required magnetic current source can be obtain as $\mathbf{M}_{s}=-\hat{\boldsymbol{\xi}} \times \mathbf{E}_{t}$ with $\hat{\boldsymbol{\xi}}$ denoting the unit normal vector pointing into the wave port (pointing from side II to I). Then we further expand the he incident electric field at the two sides of the wave port generated by the magnetic current source by the electric eigen-modes:

$$
\begin{aligned}
& \mathbf{E}_{t, \mathrm{I}}^{\mathrm{inc}}=\tilde{a}_{0}^{\mathrm{TEM}} \mathbf{e}_{0}^{\mathrm{TEM}}+\sum_{n=1}^{\infty} \tilde{a}_{n}^{\mathrm{TE}} \mathbf{e}_{n}^{\mathrm{TE}}+\sum_{n=1}^{\infty} \tilde{a}_{n}^{\mathrm{TM}} \mathbf{e}_{n}^{\mathrm{TM}} \\
& \mathbf{E}_{t, \mathrm{II}}^{\mathrm{inc}}=\tilde{b}_{0}^{\mathrm{TEM}} \mathbf{e}_{0}^{\mathrm{TEM}}+\sum_{n=1}^{\infty} \tilde{b}_{n}^{\mathrm{TE}} \mathbf{e}_{n}^{\mathrm{TE}}+\sum_{n=1}^{\infty} \tilde{b}_{n}^{\mathrm{TM}} \mathbf{e}_{n}^{\mathrm{TM}}
\end{aligned}
$$

Due to the absence of the electric surface current, thus the tangential continuity of the incident magnetic field at the wave port is valid is kept. Based on this boundary condition, we can obtain the relations between the mode expansion coefficients at the two sides of the wave port. Namely,

$$
\tilde{b}_{n}^{\mathrm{TEM} / \mathrm{TE} / \mathrm{TM}}=-\tilde{a}_{n}^{\mathrm{TEM} / \mathrm{TE} / \mathrm{TM}} \text { with } n=0, \cdots, \infty
$$

By substituting (5) into (3) and (4), we can obtain the mode coefficients of the incident electric field for a given magnetic current excitation Ms. It is expressed as:

$$
\mathbf{M}_{s}=2\left(\tilde{a}_{0}^{\mathrm{TEM}} \mathbf{e}_{0}^{\mathrm{TEM}}+\sum_{n=1}^{\infty} \tilde{a}_{n}^{\mathrm{TE}} \mathbf{e}_{n}^{\mathrm{TE}}+\sum_{n=1}^{\infty} \tilde{a}_{n}^{\mathrm{TM}} \mathbf{e}_{n}^{\mathrm{TM}}\right) \times \hat{\boldsymbol{\xi}}
$$

The mode expansion coefficients for the incident electric field can be calculated by

$$
\tilde{a}_{n}^{\mathrm{TEM} / \mathrm{TE} / \mathrm{TM}}=\int \hat{\boldsymbol{\xi}} \times \mathbf{M}_{s} / 2 \cdot \mathbf{e}_{n}^{\mathrm{TEM} / \mathrm{TE} / \mathrm{TM}} d S
$$

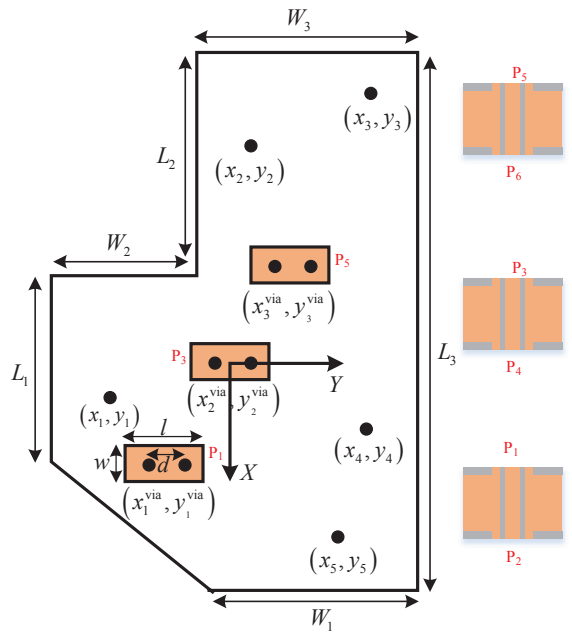

Fig. 2. Geometrical illustration of the irregular parallel plate pair with three differential via-pairs having rectangular anti-pads. The separation distance $d$ of the differential pair is $0.508 \mathrm{~mm}$, the width $w$ and length $l$ of the anti-pad are $0.762 \mathrm{~mm}$ and $1.27 \mathrm{~mm}$, respectively. The radius of the PEC rods $r_{r}$ is $0.127 \mathrm{~mm}$, the radius of the via barrel $r_{b}$ is $0.127 \mathrm{~mm}$.
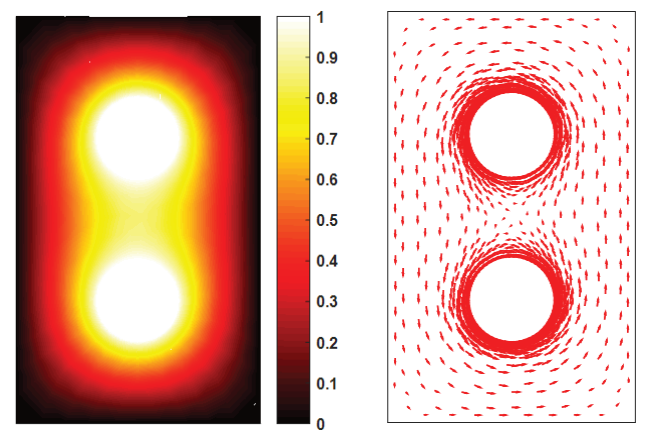

Fig. 3. Left: numerically solved common mode potential distribution $\Phi(r, \phi)$ over the circular anti-pad. Right: common mode magnetic current distribution $\mathbf{M}_{s}(r, \phi)$.

To extract the S-parameters, we assume that the excitation $\mathbf{M}_{s}$ is applied at port $j$ and the field acquired from DGTD analysis at port $i$ is $\mathbf{E}^{i}$, thus the expansion coefficient corresponding to mode $n$ is given by

$$
a_{n}^{j}=\int \mathbf{E} \cdot \mathbf{e}_{n} d S .
$$

With (8) and (7), the S-parameters for mode $n$ can be conveniently evaluated by

$$
S_{i j}= \begin{cases}\frac{a_{n}^{i}-\tilde{a}_{n}^{j}}{\tilde{a}_{n}^{j}} & i=j \\ \frac{a_{n}^{i}}{\tilde{a}_{n}^{j}} & i \neq j\end{cases}
$$

with $\tilde{a}_{n}^{j}$ denoting the incident mode expansion coefficient.

\section{DGTD FORMULATION}

To analyze the multi-layered power-ground plate-pairs by DGTD, we firstly split the computational domain of interest $\Omega$ into non-overlapping mesh cells $\Omega_{i}$. In each element, the unknowns $\mathbf{E}^{i}$ and $\mathbf{H}^{i}$ are approximated by vector edge basis 


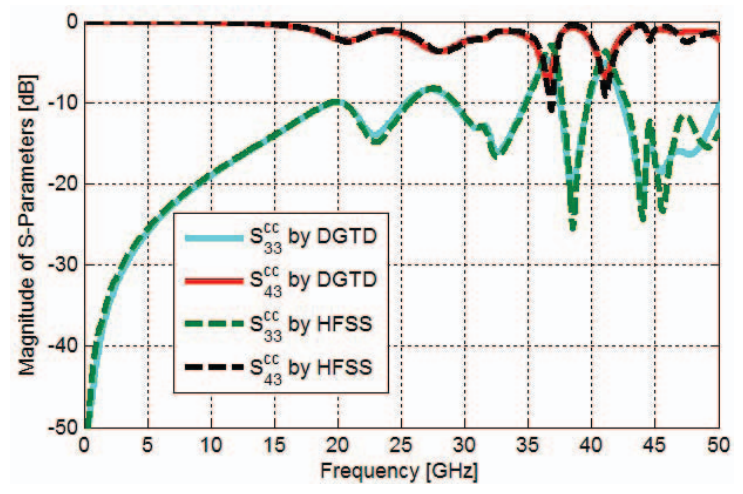

Fig. 4. The calculated common-mode S-parameters of port-pair 3 and 4 for single layer plate-pair in Fig. 2 and the results from HFSS.

functions. By conducting the Galerkin testing over the two first-order time-derivative Maxwell's equations, we can obtain the two matrix equations:

$$
\begin{aligned}
& \overline{\mathbf{M}}_{e}^{i} \frac{\partial \mathbf{e}^{i}}{\partial t}=\overline{\mathbf{S}}_{e}^{i} \mathbf{h}^{i}+ \sum_{f=1}^{n_{i}^{f}}\left(\overline{\mathbf{F}}_{e e}^{i i, f} \mathbf{e}_{f}^{i}+\overline{\mathbf{F}}_{e e}^{i j, f} \mathbf{e}_{f}^{j}\right. \\
&+\left.\overline{\mathbf{F}}_{e h}^{i i, f} \mathbf{h}_{f}^{i}+\overline{\mathbf{F}}_{e h}^{i j, f} \mathbf{h}_{f}^{j}\right)+\beta \cdot \overline{\mathbf{F}}_{e}^{i, \mathbf{M}_{s}} \\
& \overline{\mathbf{M}}_{h}^{i} \frac{\partial \mathbf{h}^{i}}{\partial t}=-\overline{\mathbf{S}}_{h}^{i} \mathbf{e}^{i}+\sum_{f=1}^{n_{i}^{f}}\left(\overline{\mathbf{F}}_{h h}^{i i, f} \mathbf{h}_{f}^{i}+\overline{\mathbf{F}}_{h h}^{i j, f} \mathbf{h}_{f}^{j}\right. \\
&\left.+\overline{\mathbf{F}}_{h e}^{i i, f} \mathbf{e}_{f}^{i}+\overline{\mathbf{F}}_{h e}^{i j, f} \mathbf{e}_{f}^{j}\right)+\beta \cdot \overline{\mathbf{F}}_{h}^{i, \mathbf{M}_{s}}
\end{aligned}
$$

where $\beta=1$ if the face $f$ is over the wave port, otherwise $\beta=0, \overline{\mathbf{M}}_{e, h}^{i}, \overline{\mathbf{S}}_{e, h}^{i}$ and $\overline{\mathbf{F}}$ are mass, stiffness and flux matrices, respectively.

\section{Numerical Results}

To validate the proposed algorithm, an irregular plate-pair including three differential via-pairs with rectangular anti-pads is benchmarked. For the common-mode analysis, the potential distribution over the rectangular anti-pad is calculated by solving the Laplace equation governed by the common-mode pertinent boundary condition. In Fig. 3 , the obtained potential distribution and the magnetic current for the common-mode are presented. As expected, the obtained results agree with the theories.

Firstly, a single-layer plate-pair is studied. The thickness of the plate-pair is $h=0.254 \mathrm{~mm}$ and the permittivity of the media is 2.2. In Fig. 4, the calculated S-parameters corresponding to the port-pair 3 and 4 are presented. For comparison, the results from HFSS are also provided. As can be seen, very good agreements are reached.

Next, the proposed algorithm is extended to investigate a four-layer power-ground plate-pair. Each layer has same thickness $\mathrm{h}=0.254 \mathrm{~mm}$. In Fig. 5 and 6, the common-mode S-parameters for port-pairs 1 and 2, and port-pair 5 and 6 are presented. To validate the accuracy of the proposed algorithm,

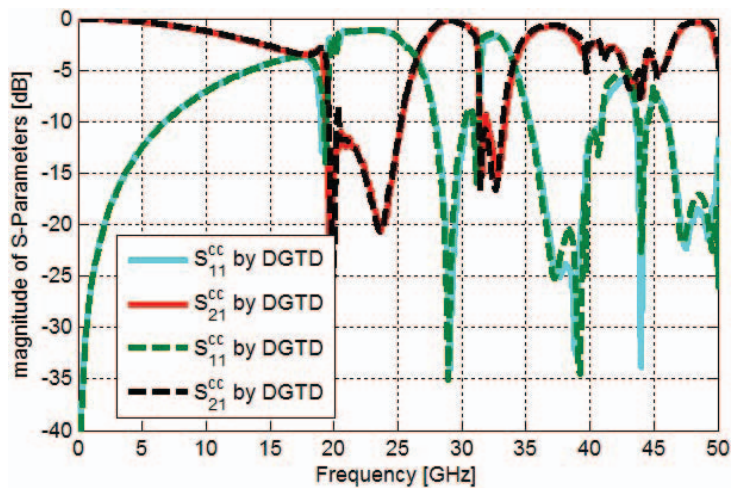

Fig. 5. The calculated common-mode S-parameters of port-pair 1 and 2 for four layer plate-pair in Fig. 2 and the results from HFSS.

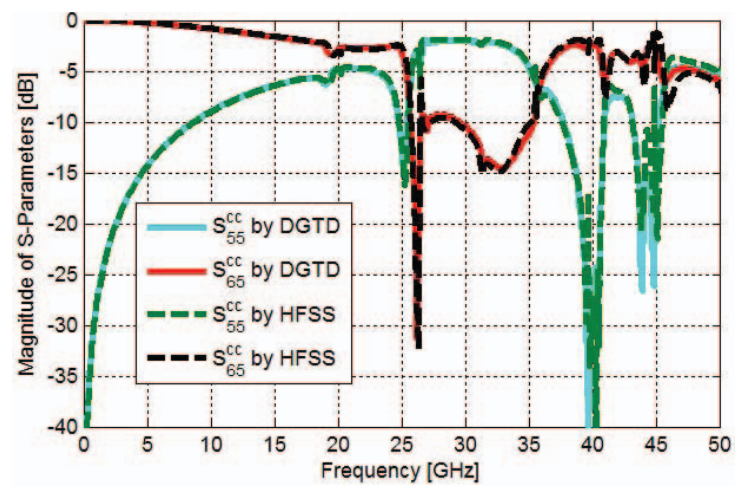

Fig. 6. The calculated common-mode S-parameters of port-pair 5 and 6 for four layer plate-pair in Fig. 2 and the results from HFSS.

the results from HFSS simulation are given. It is noted that good agreements are achieved.

\section{CONClusion}

In this work, a DGTD algorithm is developed for the analysis of power-ground planes with arbitrarily-shaped antipads. To implement the proper excitation, the wave port is employed. Furthermore, the mode pertinent S-parameter extraction method is proposed. The accuracy and feasibility of the algorithm are verified by comparing the reference results from HFSS simulation.

\section{REFERENCES}

[1] L. Tsang, H. Chen, C. C. Huang, and V. Jandhyala, "Modeling of multiple scattering among vias in planar waveguides using Foldy-Lax equations," Microw. Opt. Technol. Lett., vol. 31, pp. 201-208, Nov. 2001.

[2] Y. J. Zhang, L. H. Ren, D. Z. Liu, S. De, X. X. Gu, Y. H. Kwark, C. Schuster, and J. Fan, "An efficient hybrid finite-element analysis of multiple vias sharing the same anti-pad in an arbitrarily shaped parallelplate pair," IEEE Trans. Microw. Theory Tech., vol. 63, no. 3, pp. 883-890, Mar. 2015.

[3] Y. J. Zhang and J. Fan, "An intrinsic circuit model for multiple vias in an irregular plate pair through rigorous electromagnetic analysis," IEEE Trans. Microw. Theory Tech., vol. 58, no. 8, pp. 2251-2265, Aug. 2010.

[4] P. Li, L. J. Jiang and H. Bagci, 'Transient analysis of dispersive powerground plate pairs with arbitrarily shaped antipads by the DGTD method with wave port excitation," IEEE Trans. Electromagn. Compat., vol. 59, no. 1, pp. 172-183, Sept. 2016. 